Careful measurements using metallic glasses permit the testing of theories for the anomalous transport properties of disordered systems, notably those involving quantum effects.

\title{
Anomalous Transport in High Resistivity Metallic Glasses
}

Low-temperature transport properties perhaps demonstrate the most interesting of all the anomalous features found in amorphous metallic alloys because they represent macroscopic quantum phenomena with a rich variety of behaviours. For example, the electrical resistivity at low temperatures of some metallic glasses shows different temperature dependences (Fig. 1) originating from quantum effects. The magnetoresistance $\Delta \rho(B) / \rho-$ the dependence of the electrical resistivity $\rho$ on the magnetic field $B$ - is also anomalous at low temperatures. For instance, the magnetoresistance at 4.2 $\mathrm{K}$ is seen in Fig. 2 to be positive for Cu-La and negative for Cu-Mg.

The anomalies in electrical resistivity, magnetoresistivity and other transport properties of disordered materials arise since the electron mean path (the distance between electron collision events) becomes very short in disordered materials, of the order of the distance between atoms. In this situation, classical transport theories are no longer valid. The electrons cannot be described as independent particles and quantum corrections to the transport properties must be considered. These effects are of two types: weak localization (WL) and electronelectron interaction (EEI).

Before discussing the status of approaches for describing quantum corrections to the transport properties of metallic glasses at low temperatures, it is useful to briefly review the full range of anomalous properties.

\section{Electrical Resistivity}

The electrical properties of amorphous metals have in fact offered repeated surprises for more than 15 years. Although the temperature coefficient of resistance for a resistive metallic glass (an amorphous metallic alloy with unfilled d-bands having a high electrical resistance) is small, often a few times $10^{-4}$, the more physically interesting quantity, namely the magnitude of the temperature $T$ dependence of the electrical resistivity $d \rho / d T$ is, in contrast, often large (roughly five times larger than that of copper at room temperature for typical resistivities of amorphous metals of about $200 \mu \Omega \mathrm{cm}$ ). Moreover, the coefficient is negative from helium temperatures up to several hundreds of degrees centigrade, where relaxation effects and/or crystallization of the glass sets in. This unusual temperature dependence is the oldest reported anomaly in the electrical properties of amorphous metals.

Östen Rapp was appointed temporary professor at The Royal Institute of Technology (KTH), S-100 44 Stockholm, in 1992. He studied physics at the KTH, where he remained on the research staff after receiving his Ph.D. in 1970.

\section{Ö. Rapp}

The Royal Institute of Technology, Stockholm, Sweden

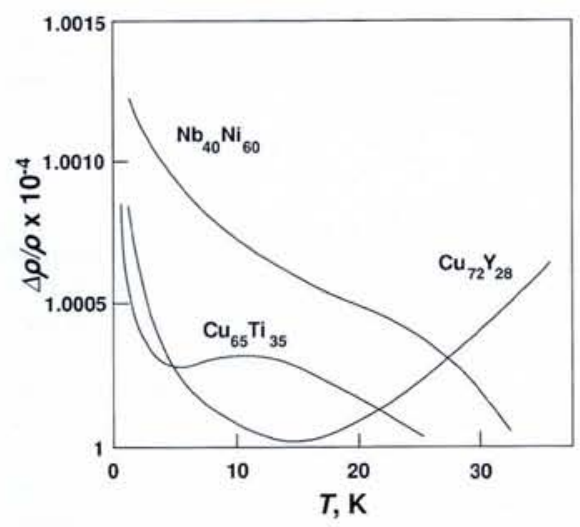

Fig. 1 - Examples of anomalous low-temperature transport properties in amorphous metallic alloys. Different types of low-temperature behaviour are observed for the electrical resistivity owing to different quantum corrections. $\rho_{o}$ is a normalization constant that varies from sample to sample.

Empirical formulations such as the Mooij correlation, with its additions and improvements [1], are useful as a summary of the phenomenon, but an understanding of the physics of the transport properties of amorphous metals with unfilled d-bands remains controversial or lacking. Some possibilities, all of which are observed to give a negative $\mathrm{d} \rho / \mathrm{d} T$, are Mott's s-d scattering model, extensions of the Ziman model for liquids, an application of the low-temperature tunnelling model, and the suggestion that quantum corrections can also give contributions at intermediate temperatures [2]. In general, one can perhaps say that the temperature dependence of the electrical resistivity of resistive metallic glasses is not as hotly debated today as it was 10 years ago because we do not have new arguments or sufficiently delicate tools for this difficult problem, and not because we have a better understanding.

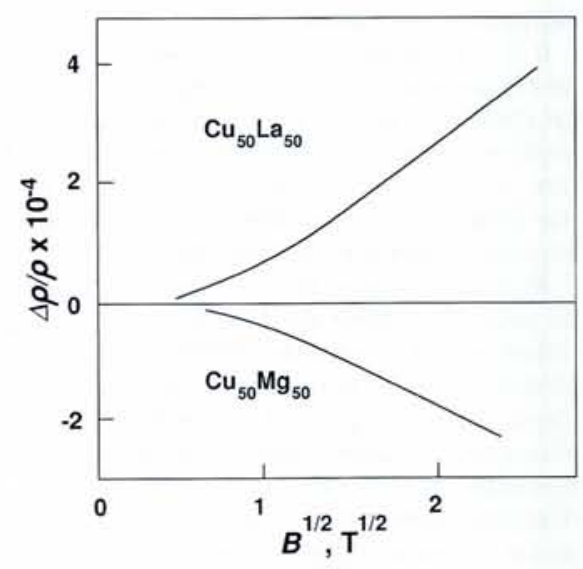

Fig. 2 - The magnetoresistance for two amorphous metals at $4.2 \mathrm{~K} . \Delta \rho / \rho$ is negative for a weak spin-orbit scattering and positive at moderate magnetic fields for a strong spinorbit scattering.

\section{Hall Effect}

Another controversial aspect of the transport properties of metallic glasses concerns the Hall effect (the creation of a voltage perpendicular to the direction of current flow when the sample is placed in a magnetic field). Electronic properties are expected to be isotropic in amorphous alloys so the Fermi surface would be spherical, albeit somewhat blurred, and the Hall coefficient should be negative. Both are observed and often quantitatively well accounted for in simple amorphous alloys with a few components. However, there are complications for amorphous alloys with unfilled d-bands. In many of these systems, the Hall coefficient is positive and sometimes shows a strong compositional dependence. Models to account for such behaviour are generally developed within one of two major groups involving either s-d hybridization or sidejump effects.

Weak localization (WL): the tendency of an electron to remain where it is rather than move freely through the material. As this tendency becomes stronger, the electron will eventually localize completely, and a metal-insulator transition occurs.

Electron-electron interaction (EEI): in contrast to weak localization, electron-electron interaction is not a single-particle effect. It arises when electron scattering becomes so frequent that interaction between scattered electron waves must be taken into account. s-d hybridization: the mixing of electron bands of s-and p-wave characters.

Side-jump effects: originate from an idea by Berger for ferromagnets according to which coupling between the spin and orbital angular momenta of the electrons could result in a sideways displacement of electrons and a positive Hall effect. These ideas have subsequently been applied to paramagnetic amorphous metals. 


\section{Superconducting Properties}

Several of the d-band amorphous metals are superconducting, and some of the superconducting properties are occasionally anomalous. One example is the upper critical magnetic field, which sometimes seems to exceed values consistent with traditional theories for disordered superconductors. Various interpretations of this effect are based on inhomogeneities, the effects of disorder, and even an inadequate analysis of experimental data.

Another, more recent, example is a strange peak in the electrical resistivity in the lower part of the superconducting fluctuation region, just above the superconducting transition temperature. Since the observation of this anomaly in amorphous superconductors, there have been several reports of similar effects in thin films, one-dimensional wires, mesoscopic loops, and in high- $T_{c}$ copper-oxide derived superconductors. Attempted explanations are based on vortexantivortex annihilation, two different effects arising from the granularity of the superconducting material, and current redistribution due to inhomogeneities [3].

\section{Probing Quantum Corrections}

Following the paper by Abrahams et al. in 1978 [4], theories developed over the next few years for the various quantum contributions to low-temperature transport phenomena [5] prompted intensive experimental work [6]. Metallic glasses represent a subcategory of those materials where quantum effects can be observed. Doped semiconductors and thin films may in many respects allow more detailed tests and illustrations. For instance, in the former, carrier concentrations can be controlled and varied over wide ranges, while in the latter, quantum corrections are much larger than in threedimensional systems, thus providing for a more straightforward separation of different effects.

Amorphous metals are three-dimensional at all temperatures since the smallest dimension of, for example, a ribbon produced by rapidly solidifying a narrow jet of molten alloy (the melt spinning process) is about $10 \mu \mathrm{m}$. This is larger than all relevant length scales such as the inelastic scattering length, even at the lowest attainable temperatures. Thus, unlike what may be the case in thin films, there are no complications from the slowly varying dimensions of the sample. However, in contrast to other threedimensional materials where WL and EEI effects are observed, amorphous metals can often form across wide concentration regions to allow a flexible and powerful study of the influence of various parameters.

The inelastic scattering time $\tau_{\mathrm{ie}}$ increases with decreasing temperature, and the elastic scattering time $\tau_{e}$ in an amorphous metal is fairly small owing to the frequent scattering from defects. One can therefore reach a low-temperature regime where $\tau_{\text {ie }} \gg \tau_{\mathrm{e}}$. The electrons undergo multiple elastic scattering with preserved phase before an inelastic, phase breaking event occurs and quantum interference effects are observed; these effects lead to the contributions from weak localization. There may also be contributions from other scattering mechanisms such as, for example, a change of spin states due to either spin-orbit scattering or magnetic scattering when magnetic impurities are present: The relevant scattering times for these two events $\left(\tau_{\mathrm{so}}\right.$ and $\tau_{\mathrm{m}}$. respectively) must therefore be considered. Furthermore, elastic scattering becomes frequent in amorphous metals and such events cannot be considered to be independent, as in Boltzmann's theory for instance; the scattered waves interfere and may cause observable electron interaction effects.

With some simplification, one can say that the electrical resistivity at low temperatures is dominated by EEI effects, while WL gives the main contribution to the magnetoresistance, at least when the temperature is not too low or the magnetic field not too high.

\section{Signatures}

The ubiquitous signature of EEI effects in the electrical resistivity is a $-T^{1 / 2}$ term at low temperatures. In amorphous alloys, this effect usually shows up below about $4 \mathrm{~K}$; it is reflected, for example, by an upturn at low temperatures of resistivity curves (as in Fig. 1). This effect is clearly revealed when the resistivity is plotted as a function of $T^{1 / 2}$. It arises owing to interference of electronelectron scattering from static inhomogeneities leading to a singularity in the electronic density of states and an additional $-T^{1 / 2}$ contribution to the electrical resistivity.

In the case of the magnetoresistance of amorphous metals at low temperatures, the WL contribution can have either sign, depending on the relative magnitudes of the inelastic and spin-orbit scattering times. When the spin-orbit scattering is weak, the dominating influence of the magnetic field is to destroy phase coherence of the electrons, thus diminishing WL; the electrical resistivity decreases with the magnetic field in this case. When the spin-orbit scattering is strong, this situation is reversed, and increases with $B$ as long as $B$ is not too large. This is demonstrated in Fig. 2 where it is seen that the dependence of the electrical resistivity on the magnetic field $\Delta \rho(B) / \rho$ at $4.2 \mathrm{~K}$ is positive for a strong spin-orbit scattering system such as $\mathrm{Cu}-\mathrm{La}$, and negative for weak spin-orbit scattering system such as $\mathrm{Cu}-\mathrm{Mg}$. A second characteristic feature is that $\Delta \rho(B) / \rho$ starts with a $B^{2}$ dependence and then reaches a region linear in $B^{1 / 2}$ at large $B$. Finally, for strong spin-orbit scattering $\Delta \rho(B) / \rho$ will eventually pass through a maximum and decrease with increasing $B$ at high magnetic fields.

Interaction effects will also influence the Hall effect and they manifest themselves as a temperature-dependent contribution with an amplitude which is twice that of the EEI contribution to the resistivity. This relation between two measured quantities provides a powerful check on the consistency of analyses.

\section{Precise analysis}

The theoretical framework for low-temperature transport anomalies based on electron-electron interactions and weak localization gives a qualitative understanding of the observed properties, and sometimes even a good quantitative description. However, a detailed analysis of the various quantum corrections is complex; to account for observations one must determine a fair number of parameters including the electrical resistivity, the diffusion constant, the magnitude and temperature dependence of $\tau_{i e}$, the magnitude of $\tau_{\mathrm{so}}$, the effective electron gyromagnetic ratio, and two EEI constants which depend on details of the studied material such as the electronic screening length and the Fermi energy. In addition, magnetic impurities will contribute from electron scattering from magnetic spins, and from phase breaking. Finally, there will be contributions from fluctuations for superconducting samples.

Even with the obvious simplifications of non-superconducting materials and negligible magnetic impurities, any analysis is difficult. It is even more demanding to accurately describe quantum corrections in 3-d materials since the effects are smaller than in 2-d systems. Approaching the precision obtained in thin films, where quantum interference effects can be used as a spectroscopy for different microscopic time scales, remains a challenge.

\section{Inventive measurements}

To deal with the complex situation found in 3-d materials, experimentalists look for simpler situations such as measurement ranges where significant simplifications can be made, or experiments where one parameter can be varied in an independent and controlled way. These efforts [6], involving great inventiveness, illustrate the richness and beauty of the field. One approach is to go to extremes of magnetic field and/or temperature where theories may be simplified (see box). Another involves systematic variation of an important parameter. For example, the spin-orbit scattering rate $1 / \tau_{\text {so }}$ can be increased by adding small amounts of a heavy element such as gold, as was first carried out by Bergmann [6] in thin films. Amorphous metals easily lend themselves to this type of experiment since a microscopically homogeneous phase can be pre-

\section{Probing Quantum Corrections at Extremes}

Quantum corrections to transport properties are probed using extremes of magnetic field and/or temperature where theories may be simplified. Methods include the use of: - very low magnetic fields, where all the contributions to the magnetoresistance follow a $B^{2}$ dependence;

- very high magnetic fields where the spin-orbit scattering rate can be determined from the change of sign of $\Delta \rho / p$;

- very low temperatures so one can reliably extrapolate to $0 \mathrm{~K}$, where theories can be greatly simplified;

- large values of $B / T$ in a region where the inelastic scattering rate $1 / \tau_{\mathrm{ie}}$ has saturated, thus allowing the contribution from electron-electron interaction to the magnetoresistance to be estimated without curve fitting or adjustable parameters. 
served over a wide range of concentrations Alternatively, one can decrease $1 / \tau_{\text {so }}$ by adding a light element such as hydrogen. These experiments are useful in helping understand the role of spin-orbit interaction.

Finally, one can study simple amorphous metals without d-electrons at the Fermi surface. These metals are often well characterised from a number of measurements, and their properties in an intermediate temperature range are usually better understood than the d-band metals.

\section{The state-of-the-art}

The state-of-the-art and current issues involved in developing quantum corrections to transport anomalies are illustrated by results plotted in Fig. 3. Fig. 3a shows that the WL contribution (proportional to $B^{2}$ ) of the magnetoresistance of amorphous $\mathrm{Cu}_{65} \mathrm{Ti}_{35}$ can be observed at temperatures as high as $85 \mathrm{~K}$ using precision measure ments. The reason is that at this temperature, a magnetic field of $10 \mathrm{~T}$ is weak since the inelastic scattering rate is large. The data are clearly in the $B^{2}$ region, with $\Delta \rho(B) / \rho=7 \times 10^{-6}[8]$.

Fig. $3 b$ plots a factor $F_{\sigma}$ which is proportional to the dominant EEI contribution to the magnetoresistance. The data are for a series of amorphous $\mathrm{Ca}-\mathrm{Al}$ alloys where the spin-orbit interaction was varied by adding small amounts of silver or gold. The results show that the EEI contribution to the magnetoresistance vanishes at large spin-orbit scattering rates [9]
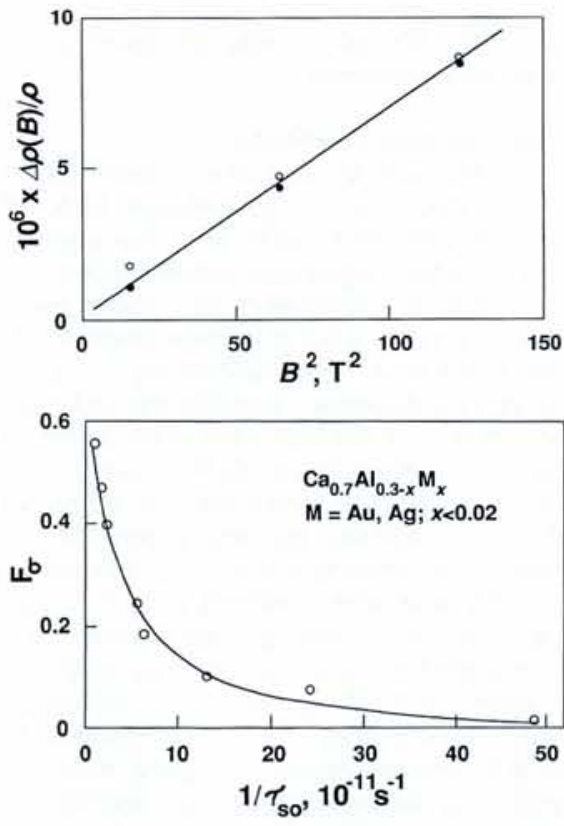

Fig. 3 - Some recent results on quantum corrections to the magnetoresistivity:

a, upper) The weak localization contribution to $\Delta \rho / \rho$ for an amorphous Cu-Ti sample of resistivity $180 \mu \Omega \mathrm{cm}$ is observed at $85 \mathrm{~K}$ to be proportional to $B^{2}$ up to $12 T$ [8].

$b$, lower) $F_{\sigma}$ is a constant multiplying the dominant EEl contribution to the magnetoresistance. The spin-orbit scattering time $\tau_{s o}$ is monitored by adding heavy elements to amorphous $\mathrm{Ca}-\mathrm{Al}$. The results show that the contribution to $\Delta \rho / \rho$ from electron-electron interactions vanishes on increasing the degree of spin-orbit scattering. From [9].

\section{Quantum Effects in Quasicrystals Raise Questions}

The magnetoresistance up to $30 \mathrm{~T}$ and 30 $K$ of icosahedral $\mathrm{Al}_{63} \mathrm{Cu}_{25} \mathrm{Fe}_{12}$ with $\rho \approx$ $4400 \mu \Omega \mathrm{cm}$ at $4 \mathrm{~K} . \Delta \rho / \rho$ scales roughly with $\rho$ when compared with observations for amorphous metals [from Klein $T$., et al., Phys. Rev. B 45 (1992) 2046]. The lines are fits to quantum corrections from WL and two EEl contributions IM. Ahlgren \& Ö. Rapp, unpublished].

The figure shows that an $\mathrm{Al}_{63} \mathrm{Cu}_{25} \mathrm{Fe}_{12}$ quasicrystal of a high degree of perfection exhibits a huge magnetoresistance $\Delta \rho / \rho$ compared with the effects observed in amorphous metals. It is about a factor 30 larger than for amorphous Cu-La (see page 102, Fig. 2) at comparable fields and temperatures. Moreover, the electrical resistivity is about $4400 \mu \Omega \mathrm{cm}$ at low temperatures, which is also about 30 times larger than for $\mathrm{Cu}_{50} \mathrm{La}_{50}$. Since contributions from weak localization (WL) and electron-electron interactions (EEI) scale with the resistivity, this is a first indication that quantum corrections to transport properties can also be observed in quasicrystals. Analysis in fact demonstrates that with reasonable values of the scattering times, WL and EEI contributions can account for a positive magnetoresistance, as shown by the lines in the figure.

These large quantum corrections in quasicrystals provide an interesting extension to the range where quantum phenomena have been usually studied. Furthermore, since quantum corrections occur in disordered systems, the results strongly suggest that quasicrystals are electronically disordered, with intense electronic scattering (small elastic scattering times $\tau_{\mathrm{e}}$ ). This feature launches another debate: how can such disorder be reconciled with the high degree of local atomic order in a quasicrystal?

These examples illustrate that careful experiments carried out using model systems allows one to test theories for the intriguing quantum effects found in disordered systems at low temperatures.

[1] Mooij J.H., Phys. Status Solidi A 17 (1973) 521; Mitzutani U., Proc. 5th Int. Conf. on Amorphous Metals, Würzburg; Eds.: S. Steeb \& H. Warlimont (North-Holland, 1985) 975; Tsuei C.C., Phys. Rev. Lett. 57 (1986) 1943. [2] Cote P.J. \& Meisel L.V., in Topics in Appl. Phys. 46, Eds.: H.J. Güntherodt \& H. Beck (Springer-Verlag, Berlin, 1981) 141; Nagel S.R., in Adv. in Chemical Physics 51, Eds.: I. Prigogine \& A.R. Rice (John Wiley, 1982) 227; Naugle D.G., J. Phys. Chem. Solids 45 (1984) 367; Howson M.A. \& Gallagher B.L., Phys. Rep. 170 (1988) 265.

[3] Lindqvist P., Nordström A. \& Rapp Ö. Phys. Rev. Lett. 64 (1990) 2941; Francavilla T.L. \& Hein R.A., IEEE Trans. Magn. MAG-27 (1991) 1039; Santhanam P., et al., Phys. Rev. Lett. 66 (1991) 2254; Crusellas M.A., Fontcuberta J. \& Pinol S., Phys. Rev. B 46 (1992) 14089; Glazman L.I., Sov. Phys.: Low. Temp. Phys. 12 (1986) 389; Rubin S., et al., Ann. der Physik: 8th Series 1 (1992) 492; Vloeberghs H., et al., Phys. Rev. Lett. 69 (1992) 1268; Vaglio R., et al., Phys. Rev. $B$ (in press).

[4] Abrahams E., et al., Phys. Rev. Lett. 42 (1979) 673.

[5] Some theoretical reviews: Altshuler B.L. \& Aronov A.G., in Electron-Electron Interaction in Disordered Systems, Eds.: A.L. Efros \& M. Pollak (North-Holland, 1985); Lee P.A. \& Ramakrishnan T.V., Rev. Mod. Phys. 57 (1985) 287; Wollhardt D., Festkörperprobleme 27 (1987) 63.

[6] Some experimental reviews: Bergmann G., Phys. Rep. 107 (1984) 1; Howson M.A. \& Gallagher B.L., Phys. Rep. 170 (1988) 265; Rapp Ö., in Disordered Systems \& New Materials, Eds:. M. Borisov, N. Kirov \& A. Vavrek, (World Scientific, 1989) 687.

[7] Bieri J.B., et al., J. Phys. F 16 (1986) 2099.
[8] Lindqvist P., J. Phys. Cond. Matter 4 (1992) 177

[9] Sahnoune A., Ström-Olsen J.O. \& Fischer H.E., Phys. Rev. B 46 (1992) 10035.

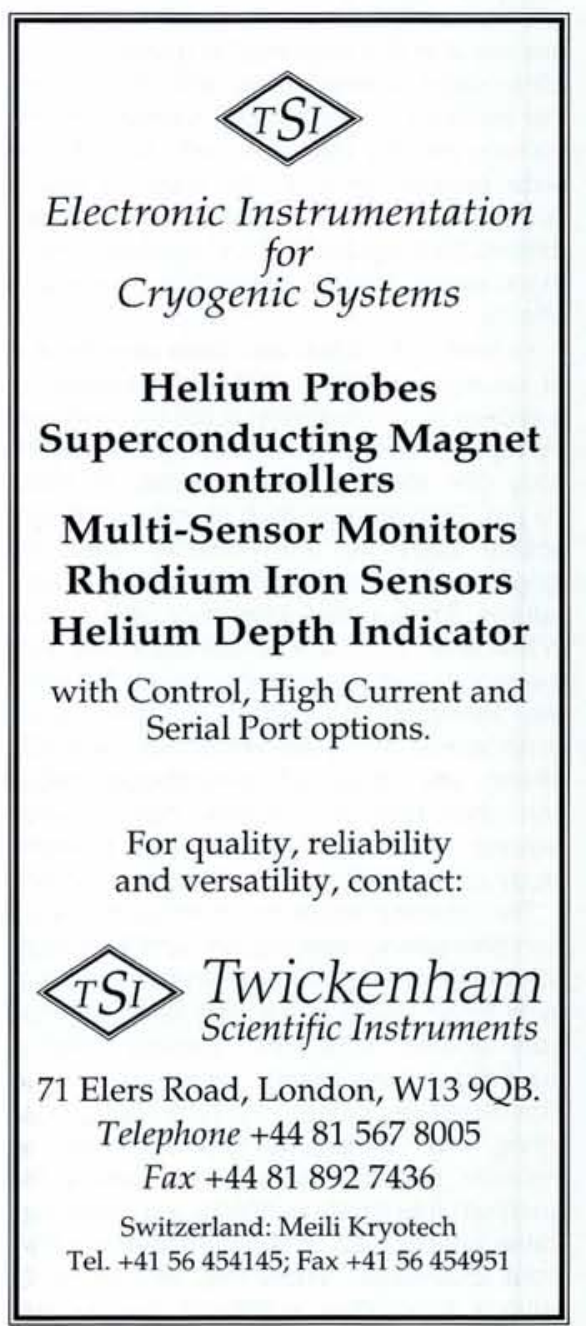

